\title{
Mapping evidence on strengthened and sustained delivery early detection and management of Hypertension and Diabetes among PLWH: a scoping review protocol
}

Abebe sorsa Badacho ( $\square$ abebe.badacho@wsu.edu.et )

Wolaita Sodo University College: Wolaita Sodo University https://orcid.org/0000-0002-1377-8298

Ozayr H Mahomed

University of KwaZulu-Natal - Howard College Campus

\section{Research Article}

Keywords: Integrated, models, Non-communicable diseases, patient outcomes, People living with HIV

Posted Date: December 30th, 2021

DOI: https://doi.org/10.21203/rs.3.rs-1213665/v1

License: (c) (i) This work is licensed under a Creative Commons Attribution 4.0 International License. Read Full License 


\section{Abstract}

Objective

The main review objective of this scoping review is to identify the evidence on the patient outcomes of integrated NCD and HIV services, the type of models used, barriers and facilitators of effective integrated services for early detection and management of hypertension and diabetes in adults PLWH in primary health care settings of Low-income countries.

Introduction:

Non-communicable diseases (NCDs) raise global public health challenges in all population groups, but the prevalence of hypertension and diabetes is highly rising among people living with HIV (PLWH). This population group requires evidencebased care for early detection and management of hypertension and diabetes. However, there is a lack of evidence regarding the impact of integrated NCD and HIV services on outcomes of PLWH, its feasibility and effectiveness of services provision and the type of models used in strengthened and sustainable services for early detection and management of hypertension and diabetes in primary health care settings. It is necessary to map and models of integrated NCD and HIV services for PLWH and its feasibility and effectiveness as well patient outcomes in a primary health care setting in lowincome countries

Inclusion criteria:

This scoping review will consider studies focusing on the model of integrated Non-communicable and HIV services for adult people living with HIV, including the type of models used, its feasibility, effectiveness and outcome of integrated services in primary health care settings. All published and grey literature will be included in the review.

Methods:

The search will be carried out using PubMed, MEDLINE with full text via EBSCO host, Google Scholar, Science Direct and Scopus, for a comprehensive search. Using MESH terms, an advanced search will be conducted and also will include grey literature through OpenGrey. This review will be restricted to studies published in English because of the feasibility of translating languages other than English. A three-step search strategy will be used, and the articles identified in the databases will be organised, and the duplicates will be removed. Two independent reviewers will review the titles and abstracts. Full texts will be imported into a bibliographic reference management system. The findings will be presented in tables and descriptive summaries.

\section{Introduction}

A preliminary search of MEDLINE, the Cochrane Database of Systematic Reviews and JBI Evidence Synthesis was conducted, and no current or underway systematic reviews or scoping reviews on the topic were identified.

More than 38 million people lived with the human immune deficiency virus (HIV) in 2019, increasing more than 8 million from 2010 globally [1]. As a result of access to antiretroviral therapy (ART) services, people living with HIV (PLWH) live longer than before [1]. Currently, more than 25.4 million PLWH are using ART services [2].

Increased access to ART services has halted an estimated 12.1 million Acquired immune deficiency syndrome (AIDS)related deaths since 2010. However, the estimated 690000 lives lost due to AIDS-related illnesses worldwide in 2019 are $39 \%$ reduction since 2010 [2]. The comorbidity of HIV and NCDs threatens to reduce global mortality due to HIV [3]. A growing number of PLWH over 50 years of age are at risk for developing chronic NCDs [4].

Exposure to Antiretroviral treatments (ART) increases the risk of hyperlipidemia and diabetes; HIV inflammatory effects, ART drug side effects, and the risk associated with increasing age can be sources for contracting NCD for PLWH[5,

Page 2/8 
6]. PLWH appears to be at a higher risk of developing NCDs. Some HIV medicines can increase blood glucose levels and lead to diabetes[6]. Some studies showed a higher incidence of NCDs among PLWH than HIV-negative patients[7].

A systematic review and meta-analysis of studies conducted in low- and middle-income countries (LMICs) showed pooled estimates for the prevalence of NCDs among PLWH; prevalence of hypertension, hypercholesterolemia, obesity, depression were $21.2 \%, 22.2 \%, 7.8 \%, 24.4 \%$, respectively, and the diabetes ranges from $1.3-18 \%$ [8].

Low-income and middle-income countries are struggling to manage growing numbers of patients with chronic noncommunicable diseases (NCDs). There have been calls for the integration of HIV and NCD services to increase efficiency and improve coverage of NCD care, although evidence of effectiveness remains unclear [9]

WHO identified a "best buy" package of 16 cost-effective, affordable, feasible, and scalable interventions in all settings[10]. The "best buys" were first selected in 2011 and were updated in 2017 based on the latest evidence of intervention impact and costs[10,11]. Cost-effective, proven interventions as a package of essential NCD interventions (WHO-PEN), with protocols and tools in primary health care for early screening and detection, were recommended [12].

The increasing burden of comorbid HIV infection and hypertension required a focus on healthcare services providing care for chronic multi-morbidities in an integrated manner [13]

The needs of PLWH with multi-morbidity are considerable because they are more vulnerable to fragmentation of care due to the involvement of multiple health professionals[14]

The main review objective of this scoping review is to identify the evidence on the integrated NCD and HIV services impact on patient outcomes, the type of models used, barriers and facilitators of effective integrated services for early detection and management of hypertension and diabetes in adults PLWH in primary health care settings.

1. To assess the patient outcome of NCD and HIV integrated services for early detection and management of hypertension and diabetes in adult people living with HIV

2. To identify the models used to integrate NCD and HIV services for early detection and management of hypertension and diabetes in PLWH?

3. To assess the feasibility of integrated NCD and HIV services for early detection and management of hypertension and diabetes among PLWH.

4. To assess the sustainability of integrated NCD and HIV services in the early detection and management of hypertension and diabetes for PLWH.

5. To identify barriers and facilitators of integrating NCD and HIV services for early detection and management of hypertension and diabetes in adult PLWH in primary health care.

\section{Review question:}

This scoping review's central question is What is known about existing literature about the effectiveness and costeffectiveness of integrated NCD and HIV services for early detection and management of common NCDs among PLWH?

This scoping review intends to answer the following sub review questions:

1. what is the impact of integrated NCD and HIV services on patient outcomes of adult PLWH?

2. What models were used to integrate NCD and HIV services to detect and manage hypertension and diabetes in PLWH in primary health care?

3. Are the integrated NCD and HIV services feasible and practical at the primary health care level? 
4. Are integrated NCD and HIV services delivered sustainably in actual-world practices?

5. What are the barriers and facilitators of integration of NCD and HIV services for PLWH?

Keywords: Barriers; facilitators; Multimorbidity; managed care; Primary health care;

\section{Eligibility Criteria}

\section{Participants}

The review will consider whose participants are adult PLWH older than 18 years with comorbidity or multi-morbidity with NCDs (i.e., adult people with HIV and other one or more non-communicable diseases). Studies focus on screening, detection management of NCDs, including diabetes, hypertension among PLWH. Those studies include participants of pregnant women will be excluded.

\section{Concept}

The concept of this review will focus on integrated management of non-communicable and HIV for PLWH used in primary care level, types of models, the impact of integrated management on patient outcomes. The integrated NCD and HIV services for PLWH will be included.

\section{Context}

\section{This review will be focused on integrated NCD and HIV services provided at primary health care level for adult PLWH in low-income countries. Studies including PLWH with multi-morbidity of one or more NCDs will be included.}

\section{Types of Sources}

This scoping review will consider both experimental and quasi-experimental study designs, including randomised controlled trials, non-randomised controlled trials, before and after studies and interrupted time-series studies. In addition, analytical observational studies including prospective and retrospective cohort studies, case-control studies and analytical crosssectional studies will be considered for inclusion. This review will also consider descriptive observational study designs, including case series, individual case reports and descriptive cross-sectional studies for inclusion. Qualitative studies will also be considered that focus on qualitative data including, but not limited to, designs such as phenomenology, grounded theory, ethnography, qualitative description, action research and feminist research.

In addition, systematic reviews that meet the inclusion criteria will also be considered, depending on the research question. Text and opinion papers will also be considered for inclusion in this scoping review.

\section{Methods}

\section{The proposed scoping review will be conducted in accordance with the JBI methodology for scoping reviews [15].}

The search strategy will aim to locate both published and unpublished studies. An initial limited search of PubMedMEDLINE and CINAHL via EBSCOhost databases was undertaken to identify articles on the topic. The text words in the titles and abstracts of relevant articles and the index terms used to describe the articles were used to develop a full 
search strategy for (see Appendix 2). The search strategy, including all identified keywords and index terms, will be adapted for each included database and/or information source. The reference list of all included sources of evidence will be screened for additional studies.

Studies published between 01 January 2000 to September 1/2021 will be included. This time framework minimal roll out of ART programs in Low and middle-income countries. Studies published in the English language will be included due to the feasibility of accessing articles published other than English and the difficulty of translating those articles. The databases to be searched include EBSCOhost with Medline complete, and CNAHIL platforms will be included. Sources of unpublished studies/grey literature to be searched include BASE for conference proceedings, reports and doctorate dissertations, Open grey and RESEARCH, and trial registers will be included.

\section{Study/Source of Evidence selection}

Following the search, all identified citations will be collated and uploaded into (EndNote 20 /2020 (Clarivate Analytics, PA, USA)) and duplicate removed. Following a pilot test, titles and abstracts will then be screened by two or more independent reviewers for assessment against the inclusion criteria for the review. Potentially relevant sources will be retrieved in full and their citation details imported into the JBI System for the Unified Management, Assessment and Review of Information (JBI SUMARI) (JBI, Adelaide, Australia) [16]. The full text of selected citations will be assessed in detail against the inclusion criteria by two or more independent reviewers. The scoping review will record and report reasons for excluding sources of evidence at a full text that do not meet the inclusion criteria. Any disagreements that arise between the reviewers at each stage of the selection process will be resolved through discussion or with an additional reviewer/s. The search results and the study inclusion process will be reported in full in the final scoping review and presented in a Preferred Reporting Items for Systematic Reviews and Meta-analyses extension for scoping review (PRISMA-ScR) flow diagram [17].

\section{Data Extraction}

Data will be extracted from papers included in the scoping review by two independent reviewers using a data extraction tool developed by the reviewers. The data extracted will include specific details about the participants, concept, context, study methods and key findings relevant to the review question/s.

A draft extraction form is provided (see Appendix XX). The draft data extraction tool will be modified and revised as necessary during the process of extracting data from each included evidence source. Modifications will be detailed in the scoping review. Any disagreements that arise between the reviewers will be resolved through discussion or with an additional reviewer/s. If appropriate, authors of papers will be contacted to request missing or additional data, where required.

A critical appraisal of individual sources of evidence: This is generally not required for scoping reviews.

\section{Data Analysis and Presentation}

The extracted data will be presented in diagrammatic or tabular form that aligns with the objective of this scoping review. Data analysis will be carried out according to the review questions. A content analysis will be conducted to classify large amounts of text into a small number of categories that represent similar meanings

\section{Declarations}

\section{Acknowledgements}


The authors intend to acknowledge the University of Kwazulu Natal, South Africa, Health Economics and AIDS research division (HEARD) giving a PhD scholarship for the primary author. Also, the author intends to acknowledge Nontobeko Precious Sikhosana, professional liberirian University of Kwazulu Natal, for professional contribution in search strategies. This scoping review is a part of a large-scale study of PhD project on strengthing and sustaining health services for early detection and management of hypertension and diabetes among PLWH in primary health centres. This scoping review will contribute PhD degree award for the primary author.

\section{Funding}

This research received no specific grant from any funding agency in the public, commercial or not-for-profit sectors. Health economics and Aids research Division (HEARD) paid salary for living expenses during the study period for the primary author.

\section{Conflicts of interest}

The authors declare that there is no conflict of interest in this project.

\section{References}

1. UNAIDS: UNAIDS epidemiological estimates of HIV , 2020. 2020.

2. (UNAIDS) UN: SEIZING THE MOMENT GLOBAL AIDS UPDATE | 2020 Tackling entrenched inequalities to end epidemics 2020 global-aids-report_executive-summary. 2020.

3. Duffy M, Ojikutu B, Andrian S, Sohng E, Minior T, Hirschhorn LR: Non-communicable diseases and HIV care and treatment: models of integrated service delivery. Tropical medicine \& international health : TM \& IH 2017, 22(8):926-937.

4. Hirschhorn LR, Kaaya SF, Garrity PS, Chopyak E, Fawzi MC: Cancer and the 'other' noncommunicable chronic diseases in older people living with HIV/AIDS in resource-limited settings: a challenge to success. AIDS (London, England) 2012, 26 Suppl 1:S65-75.

5. Nigatu T: Integration of HIV and Noncommunicable Diseases in Health Care Delivery in Low- and Middle-Income Countries. Preventing Chronic Disease: Volume 2012, 9(11).

6. NCDs and HIV: How to simultaneously address needs and enhance care

7. Coogan PF, Castro-Webb N, Yu J, O'Connor GT, Palmer JR, Rosenberg L: Active and passive smoking and the incidence of asthma in the Black Women's Health Study. American journal of respiratory and critical care medicine 2015, 191(2):168-176.

8. $\quad$ !!! INVALID CITATION !!! [8, 9].

9. Adeyemi O, Lyons M, Njim T, Okebe J, Birungi J, Nana K, Claude Mbanya J, Mfinanga S, Ramaiya K, Jaffar S et al: Integration of non-communicable disease and HIV/AIDS management: a review of healthcare policies and plans in East Africa. BMJ global health 2021, 6(5).

10. Organization WH: WHO Noncommunicable diseases country profiles 2018. . In. Geneva: WHO; 2018: 224. 
11. Organization WH: Tackling NCDs "Best buys" and other recommended interventions for the prevention and control of noncommunicable diseases. In. Geneva; 2017: 28.

12. Organization WH: Implementation tools: package of essential noncommunicable (PEN) disease interventions for primary health care in low-resource settings. Geneva: World Health Organizaiton, . Google Scholar 2013.

13. Peer N, de Villiers A, Jonathan D, Kalombo C, Kengne AP: Care and management of a double burden of chronic diseases: Experiences of patients and perceptions of their healthcare providers. PloS one 2020, 15(7):e0235710.

14. Gonçalves I, Mendes DA, Caldeira S, Nunes E: Nurse-led care management models for patients with multimorbidity in hospital settings: a scoping review protocol. JBI Evid Synth 2021, 19(8):1934-1940.

15. Peters MDJ, Marnie C, Tricco AC, Pollock D, Munn Z, Alexander L, Mclnerney P, Godfrey CM, Khalil H: Updated methodological guidance for the conduct of scoping reviews. JBI Evid Synth 2020, 18(10):2119-2126.

16. Piper C: System for the Unified Management, Assessment, and Review of Information (SUMARI). 2019 2019, 107(4):3.

17. Tricco AC, Lillie E, Zarin W, O'Brien KK, Colquhoun H, Levac D, Moher D, Peters MDJ, Horsley T, Weeks L et al: PRISMA Extension for Scoping Reviews (PRISMA-ScR): Checklist and Explanation. Ann Intern Med 2018, 169(7):467-473.

\section{Appendices}

\section{Appendix I: Search strategy}

Guidance for authors: For protocols - present a full search strategy for at least one electronic database, including planned limits, such that it can be reviewed and repeated. For systematic reviews, all search strategies should be presented.

\begin{tabular}{|c|c|c|c|}
\hline Key words used & $\begin{array}{l}\text { Date of } \\
\text { search }\end{array}$ & $\begin{array}{l}\text { Search } \\
\text { engine } \\
\text { used }\end{array}$ & $\begin{array}{l}\text { Number of } \\
\text { publication } \\
\text { retrieved }\end{array}$ \\
\hline $\begin{array}{l}((((((((\text { Effects) OR (effectiveness)) OR (outcomes)) OR (success)) OR (impact)) } \\
\text { AND (((integrated OR combined)) AND ((care OR management) AND (health } \\
\text { services delivery model)))) AND (Chronic disease OR Noncommunicable } \\
\text { disease)) AND (HIV OR Human Immunodeficiency Virus)) }\end{array}$ & $\begin{array}{l}2 \\
\text { October } \\
2021\end{array}$ & PubMed & 100 \\
\hline $\begin{array}{l}\text { outcome of Integrated Non-communicable diseases and HIV services for early } \\
\text { detection and management of NCD among PLWH }\end{array}$ & $\begin{array}{l}2 \\
\text { October } \\
2021\end{array}$ & $\begin{array}{l}\text { Google } \\
\text { scholar }\end{array}$ & 485 \\
\hline
\end{tabular}

\section{Appendix II: Data extraction instrument}

\#Only append the JBI or non-JBI data extraction instrument if the standardised tool has been modified in any way; otherwise, simply cite the tool used in the text. Any modifications made to the instrument should also be described in the text. 


\begin{tabular}{|c|c|c|c|c|c|c|c|c|c|}
\hline Authors & $\begin{array}{l}\text { Publication } \\
\text { year }\end{array}$ & $\begin{array}{l}\text { Journal or } \\
\text { other } \\
\text { sources of } \\
\text { publication }\end{array}$ & $\begin{array}{l}\text { Study } \\
\text { location } \\
\text { (country) }\end{array}$ & $\begin{array}{l}\text { Type } \\
\text { of } \\
\text { study }\end{array}$ & participants & concept & context & $\begin{array}{l}\text { Type } \\
\text { of } \\
\text { model }\end{array}$ & $\begin{array}{l}\text { Patient } \\
\text { outcome }\end{array}$ \\
\hline
\end{tabular}

